University of Nebraska - Lincoln

DigitalCommons@University of Nebraska - Lincoln

5-24-2005

\title{
Modeling of immunoglobulin uptake by N,N,N',N'- ethylenediaminetetramethylenephosphonic acid-modified zirconia particles under static and dynamic conditions
}

\author{
Sabyasachi Sarkar \\ Department of Chemical and Biomolecular Engineering, University of Nebraska, 207 Othmer Hall, 820 \\ North 16th Street, Lincoln, NE 68588, USA \\ Anuradha Subramanian \\ Department of chemical Engineering,University of Nebraska Lincoln., asubramanian2@unl.edu
}

Follow this and additional works at: https://digitalcommons.unl.edu/chemeng_biotechnology

Part of the Biochemical and Biomolecular Engineering Commons

Sarkar, Sabyasachi and Subramanian, Anuradha, "Modeling of immunoglobulin uptake by N,N,N',N'ethylenediaminetetramethylenephosphonic acid-modified zirconia particles under static and dynamic conditions" (2005). Papers in Biotechnology. 35.

https://digitalcommons.unl.edu/chemeng_biotechnology/35

This Article is brought to you for free and open access by the Chemical and Biomolecular Engineering Research and Publications at DigitalCommons@University of Nebraska - Lincoln. It has been accepted for inclusion in Papers in Biotechnology by an authorized administrator of DigitalCommons@University of Nebraska - Lincoln. 


\title{
Modeling of immunoglobulin uptake by $N, N, N^{\prime}, N^{\prime}$-ethylenediaminetetramethylenephosphonic acid-modified zirconia particles under static and dynamic conditions
}

\author{
Sabyasachi Sarkar and Anuradha Subramanian \\ Department of Chemical Engineering, University of Nebraska-Lincoln, \\ 207 Othmer Hall, P.O. Box 68588-0489, Lincoln, NE, USA \\ Corresponding author - A. Subramanian
}

\begin{abstract}
A matrix developed from $N, N, N^{\prime}, N^{\prime}$-ethylenediaminetetramethylenephosphonic acid-modified zirconia beads (further referred to as r_PEZ); $25-38 \mu \mathrm{m}$ in diameter and with a pore size of $22 \pm 3 \mathrm{~nm}$, was utilized for the separation of immunoglobulins (Igs). r_PEZ has been shown to bind to various Igs originating from a wide variety of species. To understand the mechanisms controlling the uptake of Igs by $r_{-} P E Z$, static protein uptake experiments were carried out. The protein uptake profiles were further modeled with a kinetic rate constant model. Individual studies were undertaken for human immunoglobulin A, G and M (HIgA, HIgG and HIgM). The kinetic rate constant model indicated that HIgG binding to $r_{-} P E Z$ was more favorable than its disassociation. The equilibrium rate constants were found to decrease with increasing concentration. The effect of continuous loading in a packed bed system utilizing r_PEZ matrix was evaluated by carrying out frontal studies, using different feed concentrations and linear velocities. The breakthrough profiles obtained for the uptake of HIgG were modeled with the pore diffusion model. The model was found to best describe the breakthrough profiles obtained at a feed concentration of $2.0 \mathrm{mg}$ of HIgG per milliliter. The NTU for the packed bed was found to be equal to 2 .
\end{abstract}

Keywords: zirconia, immunoglobulins, pseudo-affinity separations, modeling

\section{Introduction}

The purification of biomolecules is an important problem in downstream bioprocessing [1-9]. Economics, efficiency and practicality are some of the constraints that dictate the search for novel chromatographic supports and methodologies that offer novel selectivity or overcome the shortcomings of existing supports. Zirconia based supports, particles with thermal and mechanical stability [6], have the potential to offer both. Our previous studies have established the usefulness of $N, N, N^{\prime}, N^{\prime}-$ ethylenediaminetetramethylenephosphonic acid-modified zirconia in the separation of immunoglobulins from complex mixtures [7]. Research-based prediction of mass transport, biological activity behavior, kinetic and thermodynamic parameters that impact protein retention and separation are essential for the integration of chromatographic-based unit-operations into the purification scheme [10-16]. A quantitative or qualitative knowledge of the parameters involved in the transport of biomolecules in a chromatographic system is needed before improvements may be designed. The determination of the rate of uptake or binding of the molecules is an essential part of the information required for the modeling of the system.

Various theories have been developed to describe the binding mechanism. The most rigorous being the general mass transfer mechanism [12, 17, 18]. Suitable mathematical models have been postulated to describe and analyze the transport of proteins and solutes in porous beaded matrices and, the protein uptake from a finite medium. The kinetic rate constant model $[15,16]$ and the film and 
pore diffusion model and its variations [16, 19-21] were employed to approximate the protein uptake profiles in a finite medium. Dynamic breakthrough profiles were approximated by model equations as outlined elsewhere $[21,22]$. The adsorption phenomena were attributed due to the combined effects of solute transport and adsorption. The relevant transport equations were either analytically or numerically solved after suitable approximations and assumptions were made about the rate limiting factors driving the adsorption phenomena [23-25].

Application of transport-model equations require an estimate of the rate coefficients and equilibrium constants or require the determination of dimensionless parameters like the Sherwood or Peclet number. Under most circumstances the information required for calculating such parameters are not available. It is however, possible to first calculate these parameters and then proceed on with the modeling, using pulse injection techniques. Pulse techniques in conjunction with Laplacian transformation and statistical analysis can be used to solve the transport equations [25-27] and further obtain the transport parameters.

Our goal was optimize the chromatographic perfor-

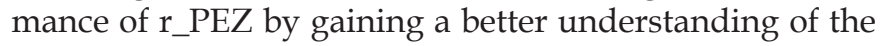
solute transport under dynamic conditions and in a finite medium. In this paper, protein uptake studies by r_PEZ in a finite medium and under dynamic conditions were undertaken to better understand the interaction of human immunoglobulins (Igs) with the r_PEZ. Experimentally obtained profiles were compared to the profile predicted by the kinetic rate constant model. The dynamic breakthrough profiles obtained from frontal analysis were approximated and compared to the profile predicted by the kinetic rate constant model $[15,16]$; with the anticipation that these engineering criteria would enable us to better understand the performance of $\mathrm{r} \_\mathrm{PEZ}$ in bioseparations.

\section{Material and methods}

\subsection{Reagents}

All chemicals were of analytical-grade or better. Sodium chloride was purchased from Fischer Scientific (Hanover Park, IL, USA). $N, N, N^{\prime}, N^{\prime}$-Ethylenediaminetetramethylenephosphonic acid (EDTPA) was purchased from TCI America (Portland, OR, USA). Bovine serum albumin (BSA), pure human immunoglobulin G (HIgG), all horseradish peroxidase conjugated anti-immunoglobulins used for ELISA were obtained from Sigma Chemical Company (St. Louis, MO, USA). All proteins were used without further purification. Human immunoglobulin A (HIgA) and human immunoglobulin $\mathrm{M}$ (HIgM) were purchased from Jackson Immunoresearch (West Grove, PA, USA).

Immulon II microtiter plates were purchased from Fisher Scientific (Hanover Park, IL, USA). Affinity purified goat anti-mouse (whole molecule) immunoglobulins and goat anti-mouse immunoglobulins conjugated to horseradish peroxidase (HRP) were purchased from
Sigma Chemical Company (St. Louis, MO, USA). o-Phenylenediamine- $2 \mathrm{HCl}(\mathrm{OPD})$ tablets were purchased from Abbott Laboratories (Chicago, IL, USA). Pre-cast NuPage $4-12 \%$ Bis-Tris gels were purchased from Invitrogen (Carlsbad, CA, USA). A Genesys ${ }^{\mathrm{TM}} 5$ model from Spectronic Instruments UV-vis spectrophotometer (Rochester, NY, USA) was used to record the adsorption measurements. A bench top microcentrifuge (Eppendorf Centrifuge 5415C) was used to sediment the r_PEZ particles for batch experiments.

\subsubsection{Support matrix preparation}

Colloidal zirconia was spray dried to yield zirconia particles, which were further classified, modified with EDTPA and characterized as reported elsewhere [28]. EDTPA-modified zirconia particles will be referred to as r_PEZ in this manuscript. $r_{-}$PEZ particles were packed into a $0.46 \mathrm{~cm}$ i.d. $\times 5.0 \mathrm{~cm}$ length analytical column, and supplied by ZirChrom (Anoka, MN, USA).

\subsection{Ligand binding isotherms}

Batch experiments conducted to determine the equilibrium binding capacity of r_PEZ for HIgG was done as described elsewhere [28]. Briefly, microfuge tubes filled with the same and known volumes of equilibrated and wet r_PEZ beads were loaded with constant volumes of HIgG solutions with different stock concentrations. Samples were allowed to equilibrate for $24 \mathrm{~h}$ and the resultant supernatant concentration measured spectrophotometrically at $280 \mathrm{~nm}$. Amount of HIgG bound was determined via mass balance.

Independent ligand binding isotherms were also determined for the binding of HIgA and HIgM to r_PEZ.

\subsection{Batch kinetic studies}

The rate of adsorption of proteins to $r$ PEZ beads were determined at different protein concentration in small batch experiments. Four hundred microliters of $50 \%$ (v/ v) slurry of r_PEZ beads were transferred into $3 \mathrm{ml}$ plastic tubes to yield approximately $200 \mu \mathrm{l}$ of beads. The beads were allowed to settle for at least $5 \mathrm{~min}$ and the liquid overlay was pipetted off after centrifuging for $5 \mathrm{~min}$ at $8000 \mathrm{rpm}$. Stock solutions of HIgG, HIgA and HIgM were prepared with appropriate dilutions. Prior to their use, HIgA and HIgM stock solutions obtained from suppliers were diafiltered and buffer replacement carried out with the LB, using Millipore's Centricon YM-10 (Bedford, MA, USA). The feed concentrations investigated for $\mathrm{HIgG}$ were 1,5 and $10 \mathrm{mg} / \mathrm{ml}$. Those for HIgA and HIgM were 0.46 and $1.84 \mathrm{mg} / \mathrm{ml}$ and 0.184 and $0.92 \mathrm{mg} / \mathrm{ml}$, respectively. Two milliliters of stock solution was introduced into the prepared r_PEZ beads at $4{ }^{\circ} \mathrm{C}$ and placed on an end-toend rotator. Ten microliters of aliquots were drawn at 0 , $0.5,1,5,10,20,30,45,60,120,240$ and $1440 \mathrm{~min}$. Stock concentration was drawn for 0 min aliquot. Experiments were performed in duplicate. Protein concentration was 
measured in the aliquots and the amount bound was found by mass balance. HIgG in the aliquots was measured by detecting the absorbance at $280 \mathrm{~nm}$. For HIgA and HIgM their respective ELISAs were performed. Data was presented as normalized concentration, $C / C_{0}$ (aliquot/supernatant concentration against feed concentration) versus time.

\subsection{Chromatography}

The chromatographic system consisted of a Chrom Tech (Apple valley, MN, USA) Iso-2000 isocratic pump in conjunction with an online Model 783 Spectroflow spectrophotometer (Ramsey, NJ, USA), which was used mainly as an indicator, and an SRI (Torrance, CA, USA) PeakSimple Model 203, single channel serial port online data acquiring system. The absorbance of the fractions was then measured using the spectrophotometer.

All buffer solutions were filtered through ChromTech's Metal-Free solvent (type A-427) $10 \mu \mathrm{m}$ ultra high molecular weight polyethylene (UHMWPE) membrane filter at the time of use.

All column experiments were performed with a $0.46 \mathrm{~cm} \times 5.0 \mathrm{~cm}$ (diameter $\times$ length) analytical column packed with approximately $30-100 \mu \mathrm{m}$ diameter zirconia beads.

\subsection{Dynamic studies}

Zirconia packed column's performance was evaluated by determining the breakthrough curves of HIgG at various flow rates and feed concentration. In all cases pure HIgG dissolved in Loading Buffer (4 mM EDTPA, $20 \mathrm{mM}$ MES, $50 \mathrm{mM} \mathrm{NaCl}, \mathrm{pH} 5.5$ ) to obtain feed concentrations of $0.5,2.0,5.0$ and $10.0 \mathrm{mg} / \mathrm{ml}$. Protein solution to be used as feed was kept in a chilled reservoir and introduced to the system continuously via the multi channel valve. Linear velocities of 3.01, 6.02 and $12.04 \mathrm{~cm} / \mathrm{min}$ were investigated. Aliquots of the outlet stream were collected and their protein content measured at $280 \mathrm{~nm}$. In all cases the protein solution was allowed to saturate the column till the flow through protein concentration reached $75-80 \%$ of the feed concentration. At the end of the loading process the proteins were eluted from the column using Elution Buffer (4 mM EDTPA, $20 \mathrm{mM}$ MES, $1 \mathrm{M} \mathrm{NaCl}$ ) and protein content measured. Zero time was marked as the time when the valve was switched from the Loading Buffer to the feed solution. Data was plotted as normalized concentration, $C / C_{0}$, of outlet protein concentration against the maximum protein concentration obtained in an aliquot; by normalized time, $T / T_{\max }$.

\subsection{Determination of HIgG, HIgA and HIgM by ELISA}

The concentrations of the Igs were determined by an ELISA procedure as outlined elsewhere [28]. Individual ELISA was carried out to determine the concentration of each species of immunoglobulins.

\subsection{Modeling and simulation}

Kinetic rate constant model equations $[15,16,19]$ were solved using a program written in MATLAB [29]. The pore diffusion rate-limiting model was used to approximate the dynamic profiles. The parameters were optimized by least squares minimization using the constrained optimization routine LSQCURVEFIT.

\section{Results}

\subsection{Ligand binding isotherms}

The maximum binding capacity $\left(Q_{\max }\right)$ and the dissociation $\left(K_{\mathrm{d}}\right)$ constant were determined from the batch isotherm data as described earlier [28]. The $Q_{\max }$ and $K_{\mathrm{d}}$ values for $\mathrm{HIgG}$ were found to be $55 \mathrm{mg} / \mathrm{ml}$ and $0.7 \mathrm{mg} / \mathrm{ml}$ for $r_{-} P E Z$ beads. Following a similar approach, the $Q_{\max }$ and $K_{d}$ values for HIgA were determined to be $18.98 \mathrm{mg} /$ $\mathrm{ml}$ and $0.01 \mathrm{mg} / \mathrm{ml}$, and that for HIgM was found to be $0.845 \mathrm{mg} / \mathrm{ml}$ and $2.486 \mathrm{mg} / \mathrm{ml}$ for r_PEZ beads. The inability to maintain both HIgA and HIgM at concentrations greater than $3 \mathrm{mg} / \mathrm{ml}$, limited our construction of reliable isotherms for these molecules.

\subsection{Kinetic uptake of Immunoglobulins under static conditions}

Small-scale batch experiments were conducted to determine the rate of uptake of HIgG, HIgA and HIgM by $\mathrm{r}_{-}$ PEZ beads from a feed solution containing Igs at various feed concentrations $\left(C_{0}\right)$. Figure $1 \mathrm{a}-\mathrm{c}$ shows the rate of disappearance of HIgG, HIgA and HIgM from the solution, respectively. Analysis of HIgG was done by measuring its respective absorbance at $280 \mathrm{~nm}$. The HIgA and HIgM concentration at different time points were estimated by their specific ELISA assays as reported elsewhere [26]. As all experiments were carried out in a closed system, it was assumed that the all Ig not measured in the solution had bound to the support.

Maximum HIgG retention by r_PEZ was observed at $24 \mathrm{~h}$ with $60-95 \%$ disappearance of protein from solution from an initial HIgG concentration of $10 \mathrm{mg} / \mathrm{ml}$ and $1 \mathrm{mg} / \mathrm{ml}$, respectively (Figure 1a). Values did not change appreciably after the 240 min time-point. The largest drop in the percentage disappearance of HIgG, i.e. greatest adsorption rate, occurs within the first $5 \mathrm{~min}$ of the batch experiment. Fifty percent of the adsorption occurs roughly after $6 \mathrm{~min}$ from the start of the experiment for a feed concentration of $1 \mathrm{mg} / \mathrm{ml}$. By $25 \mathrm{~min}$ approximately $80 \%$ of total binding has occurred. According to experimental data, $90 \%$ of the IgG has been adsorbed by the end of $50 \mathrm{~min}$. A $50 \%$ adsorption $\left(C / C_{0}=0.5\right)$ was attained at 76 and $870 \mathrm{~min}$ for HIgG concentrations of 5 and $10 \mathrm{mg} / \mathrm{ml}$, respectively.

According to experimental data, $90 \%$ of the HIgA is adsorbed by the end approximately $1400 \mathrm{~min}$ (data point not shown). A $50 \%$ adsorption was not attained for the 



Figure 1. (a) Batch kinetic uptake of human immunoglobulin G to r_PEZ beads at different concentrations. The procedure is mentioned in the materials and methods section. Maximum binding capacity and the dissociation constant of HIgG for the column was taken as $55 \mathrm{mg} / \mathrm{ml}$ and $0.7 \mathrm{mg} / \mathrm{ml}$, respectively. Kinetic rate constant model was used for the system and the values of the rate constants were determined by least square fit. $C^{\prime}$ indicates the dimensionless protein concentration after 50 min has elapsed from the start of the experiment. $C^{*}$ represents the disappearance of $50 \%$ of the initial feed concentration. Representation is done for one concentration $(1 \mathrm{mg} / \mathrm{ml})$ for clarity purposes only. (b) Batch kinetic uptake of immunoglobulin A to $\mathrm{r}_{-}$PEZ beads at different concentrations. The procedure is mentioned in the materials and methods section. Kinetic rate constant model was used for the system and the parameter values of the model were determined by least square fit. Maximum binding capacity and the dissociation constant of HIgA for the column was found to be $8.7 \mathrm{mg} / \mathrm{ml}$ and $0.29 \mathrm{mg} / \mathrm{ml}$, respectively. (c) Batch kinetic uptake of human immunoglobulin M (HIgM) to r_PEZ beads at different concentrations. Kinetic rate constant model was used for the system and the parameter values of the model were determined by least square fit. Maximum binding capacity and the dissociation constant of HIgM for the column was found to be $3.8 \mathrm{mg} / \mathrm{ml}$ and $0.055 \mathrm{mg} / \mathrm{ml}$, respectively.

higher feed concentration. According to experimental data, $90 \%$ of the solute has been adsorbed by the end of $180 \mathrm{~min}$. A $50 \%$ adsorption was attained at $200 \mathrm{~min}$ for HIgM concentration of $0.92 \mathrm{mg} / \mathrm{ml}$.

The experimental data for the protein adsorption obtained under static conditions was approximated using the "kinetic rate constant model", discussed in detail elsewhere $[16,17]$. The only unknown parameter was the forward rate contact $\left(k_{1}\right)$, where as the isotherm parameters $K_{\mathrm{d}}$ and $Q_{\max }$ determined from static binding experiments were used [28] and the reverse rate constant $\left(k_{2}\right)$ was equated to $K_{\mathrm{d}} k_{1}$. Simulations were performed with a variety of values of the unknown parameter $k_{1}$ and the value that gave the best fit of the experimental data was reported. The agreement between the experimental data and the simulation is shown in Figure 1ac. Open circles, stars and open rectangles depict experimental data and solid lines the model prediction obtained after least squares minimization. The various values of the parameters as determined by the optimized model are as indicated in Table 1. For the uptake of HIgG by $r_{-}$ PEZ, a $k_{1}$ value of $0.0242,0.0025$, and $0.0028 \mathrm{ml} / \mathrm{mg} \mathrm{min}$ was obtained at a $C_{0(\mathrm{HIgG})}$ values of 1,5 and $10 \mathrm{mg} / \mathrm{ml}$, respectively. 
Table 1. Kinetic rate constant model was used to determine the lumped forward $\left(k_{1}\right)$ and backward $\left(k_{2}\right)$ reaction rate constant. Individual experiments were done in duplicate

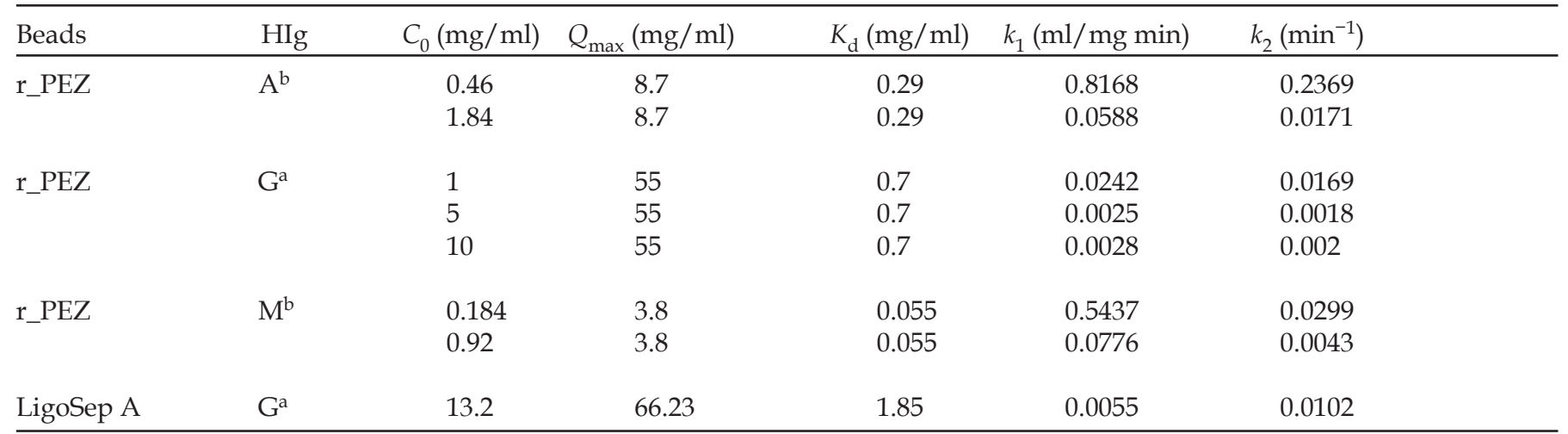

a Concentration determined by measuring respective sample absorbance at $280 \mathrm{~nm}$.

${ }^{\mathrm{b}}$ Concentration determined by respective ELISAs.

However for HIgA and HIgM, it was found after multiple attempts, that the model was unable to predict the experimentally derived profile. Hence, the procedure of unconstrained (referred further to as 'free') and constrained (referred further to as 'restricted') optimization was utilized to obtain the parameters for the best fit of the data. Free optimization was carried out on batch kinetic experiments performed for HIgG and the values obtained for $Q_{\max }$ and $K_{\mathrm{d}}$ were compared with the ones found experimentally from isotherm data. It was found that they did not differ significantly; this procedure was used to determine the $Q_{\max }$ and $K_{\mathrm{d}}$ values for HIgA and HIgM from their experimental batch kinetic data. There after restricted optimization was utilized to determine the values of the respective $k_{1}$ and $k_{2}$ values.

Figure $1 b$ and $c$ show the best fit profiles obtained for HIgA and HIgM batch kinetic data for two different feed concentrations. The open circles indicate experimental data and the solid lines the model prediction. Constrained optimization determined the $Q_{\max }$ and $K_{\mathrm{d}}$ values as 8.7 and $0.29 \mathrm{mg} / \mathrm{ml}$ for HIgA and 3.8 and $0.055 \mathrm{mg} / \mathrm{ml}$ for HIgM to r_PEZ, respectively and the values are summarized in Table 1.

Table 1 lists the values of $k_{1}$ and $k_{2}$ obtained for each Ig species, as a function of feed concentration. In general, the values of $k_{1}$ decreased with an increase of feed concentration.

\subsection{Frontal analysis}

The dynamic binding of HIgG to r_PEZ was monitored experimentally by breakthrough analysis, at different feed concentrations and linear velocities. Figure $2 \mathrm{a}$ and $\mathrm{b}$ depict representative breakthrough profiles obtained for HIgG at a feed concentration of 2 and $5 \mathrm{mg} /$ $\mathrm{ml}$, respectively. Separate breakthrough profiles were generated at three different linear velocities of 3.01, 6.02 and $12.04 \mathrm{~cm} / \mathrm{min}$, respectively. For a HIgG feed concentration of $2.0 \mathrm{mg} / \mathrm{ml}$, a $10 \%$ breakthrough was observed 17, 0.5 and $0.07 \mathrm{~min}$ at linear velocities of 3.01, 6.02 and $12.04 \mathrm{~cm} / \mathrm{min}$, respectively. A $80 \%$ breakthrough in col- umn capacity was obtained at 18, 3.4 and $1.4 \mathrm{~min}$, respectively at linear velocities of 3.01, 6.02 and $12.04 \mathrm{~cm} / \mathrm{min}$, respectively. We have obtained similar breakthrough profiles at other HIgG feed concentrations (data not shown).

The experimentally obtained breakthrough profiles were approximated by the various models available in the literature [21]. The kinetic rate constant model [15]

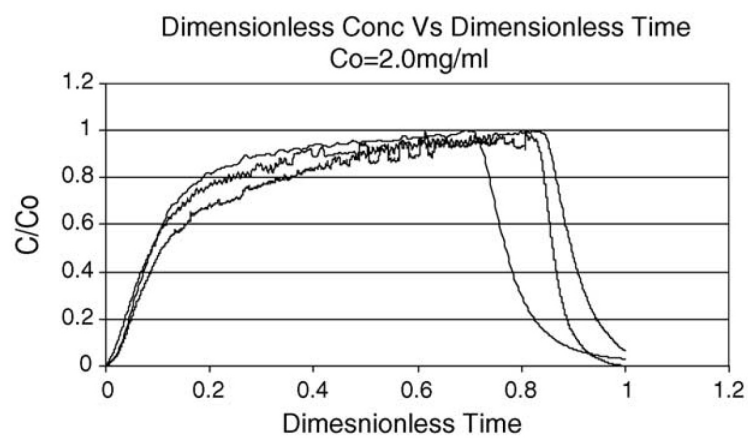

(a)
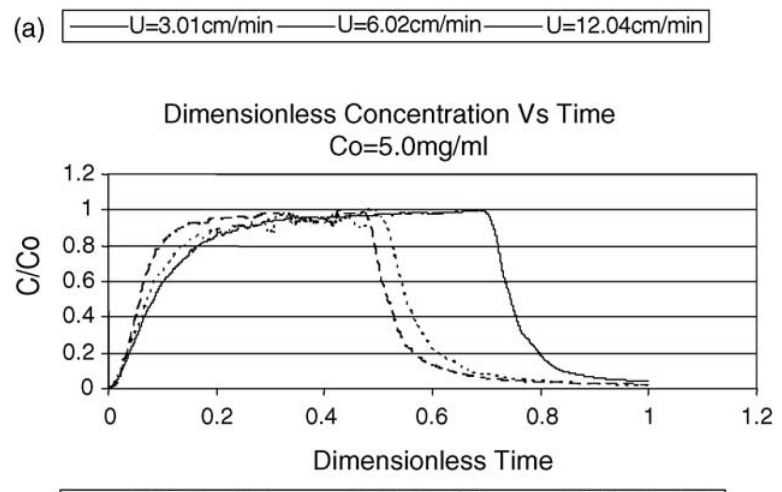

(b) $-U=3.01 \mathrm{~cm} / \mathrm{min} \cdots \cdots \cdot \mathrm{U}=6.02 \mathrm{~cm} / \mathrm{min}-\cdots \mathrm{U}=12.04 \mathrm{~cm} / \mathrm{min}$

Figure 2. (a and b) Breakthrough curves obtained for the dynamic uptake of HIgG to a packed analytical column $(0.46 \mathrm{~cm}$ i.d. $\times 5 \mathrm{~cm})$ of r_PEZ beads. Particle diameter was in the range of 3-30 $\mu \mathrm{m}$. Column was equilibrated with LB and then fed with HIgG dissolved in LB at a concentration of $2 \mathrm{mg} / \mathrm{ml}$ and $5 \mathrm{mg} / \mathrm{ml}$, respectively. The time was made dimensionless by normalizing it with respect to total time of operation. The initial time has been taken as the response time for this plot for presentation purposes only. 

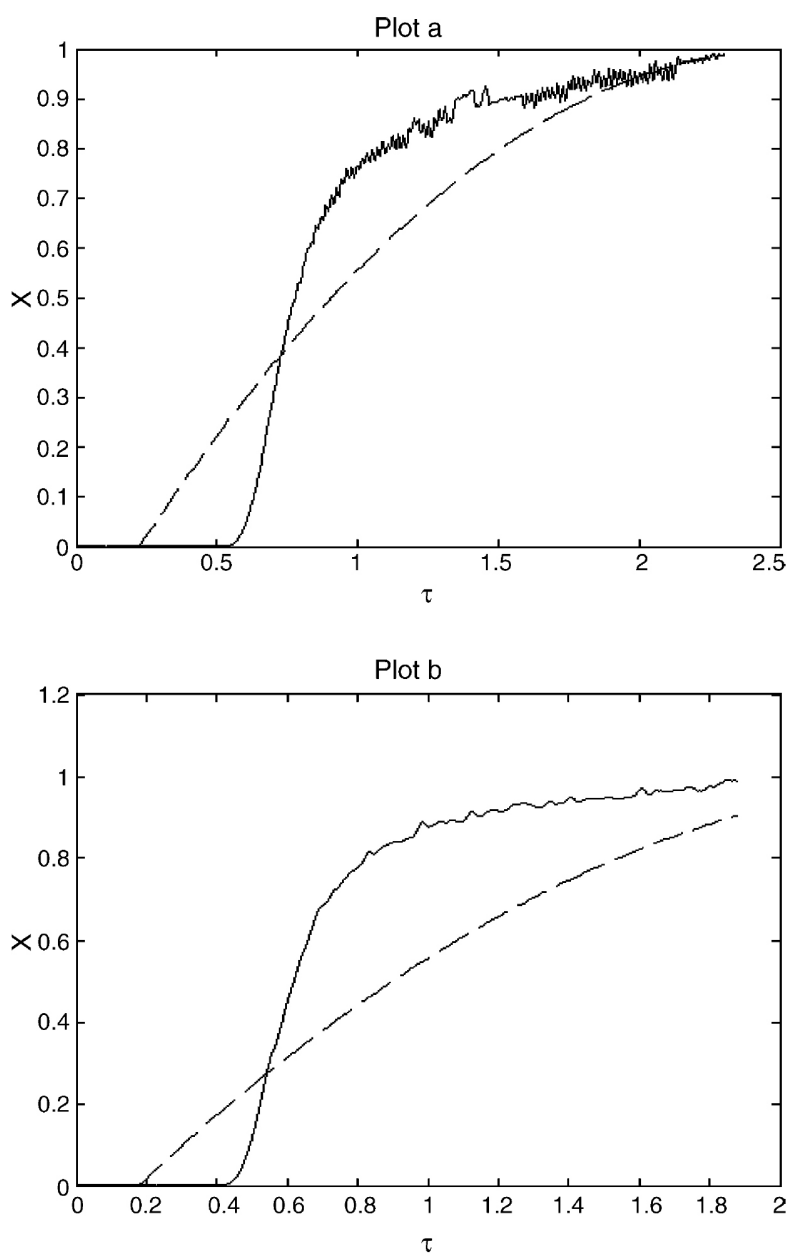

Figure 3. (a and b) Dynamic profiles plotted and modeled for individual linear velocities presented in Figure $2 \mathrm{a}$ using the pore diffusion model. Data was fitted by least squares optimization. All plots were obtained using a feed concentration of $2.0 \mathrm{mg} / \mathrm{ml}$ of HIgG. Parts (a) and (b) were obtained for linear velocities of 3.01 and $6.02 \mathrm{~cm} / \mathrm{min}$, respectively. The $N_{\text {pore }}$ value for the system was obtained to be 2 .

was unable to approximate the breakthrough profiles obtained in our study and was hence not pursued further. Other relevant model equations were used and the mathematical expression governing the pore diffusion model [21] gave a satisfactory fit to the experimental breakthrough profiles. The model prediction and the experimentally obtained breakthrough profiles are as Figure 3a and $b$. The best fit of pore diffusion model equation to the data in Figure $3 a$ and $b$ gave a rounded off value of $N_{p}$ equal to 2 .

\section{Discussion}

The long term goal of our research effort is to better understand the rate and mechanism of solute binding and transport in r_PEZ. The objective of this study, which is the next step in achieving our long term goal, is to further understand the kinetic parameters that govern the interaction under static and dynamic conditions. We seek to put forth model equations and identify mass transfer parameters relevant for a preparative scale chromatographic separation with r_PEZ. Our previous studies have shown that the binding of Igs to $\mathrm{r} \_$PEZ can be modeled with a pseudo-Langmuir isotherm [28]. Additionally it has been shown that the binding is not adversely impacted by temperature. The kinetic rate constant model; which can be modified suitably to include different adsorption rate equations without making major differences to the final form, was employed to approximate the experimentally obtained protein uptake profiles. It was found that the $Q_{\max }$ and $K_{\mathrm{d}}$ values obtained by 'free optimization' process, as described earlier, was in good agreement with those determined experimentally for HIgG data. Hence, we have used this technique to approximate the protein uptake profiles for HIgA and HIgM.

The values of the forward rate constant $\left(k_{1}\right)$ were found to decrease with increasing feed concentration as can be seen from Table 1. There is a corresponding decrease in the reverse rate constant $\left(k_{2}\right)$ also, which is implied in the definition. For individual Igs the $k_{1}$ value for comparable concentrations $(1 \mathrm{mg} / \mathrm{ml}$ of Ig) is largest for HIgM. HIgM is a tertiary molecule (pentamer) that is more bulky than the HIgG molecule, with multiple binding moieties. It adheres to the binding sites more strongly as a result $k_{1}$ values tend to be higher. It is predicted that the $k_{1}$ value for HIgA for a feed concentration of $1 \mathrm{mg} / \mathrm{ml}$ would be higher than that for HIgG using the same arguments. This maybe deduced from the trend in the $k_{1}$ values as shown in Table 1.

Higher values of $k_{1}$, when compared to $k_{2}$, for Ig adsorption to $r_{-} P E Z$ indicate that the mechanism of the adsorption of Igs are favored over desorption. This phenomenon is apparent by the presence of tailing sections in elution profiles [26]. Under dynamic loading conditions, the rate of adsorption is observed to be higher than that during desorption, as evident in Figure 2a and b. As both $k_{1}$ and $k_{2}$ are lumped coefficients it can be only inferred from the trend in their values for $r_{-} P E Z$ that the mechanisms responsible for mass transfer, decrease with increasing Ig concentration. This may be due to the spatial exclusion exerted by the adsorbed biomolecule and its impact on pore diffusive fluxes.

Langmuir isotherms in conjunction with the kinetic rate constant model have been reported to be able to successfully model both batch kinetic and frontal experiments [15]. However, the above-mentioned model did not provide a satisfactory approximation to the dynamic breakthrough profiles obtained in this study. The possible reason maybe that the mobility of HIgG through the pores of $r_{-} P E Z$ is the rate limiting process and aforementioned model does not consider it explicitly [15]. The adsorption of HIgG maybe favorable only at the outer pe- 
ripheral surface of the r_PEZ particles, making liquid film mass transfer the dominant mechanism in the initial phase of adsorption. However in the later phase, adsorption takes place in the interior of the beads owing to the unavailability of free sites at the surface, and this process maybe slower than the initial surface adsorption rate. In a parallel study, we have used FITC-labeled HIgG and its subsequent visualization by confocal laser scanning microscopy (CLSM) to visualize the distribution of binding sites through out the cross-section [30]. Our results show a uniform FITC signal throughput the cross section at $\mathrm{HIgG}$ loadings of $5.0 \mathrm{~m} \mathrm{~h} \mathrm{IgG/ml}$ or higher (data not included).

As a next step, we have used the "pore diffusion model" to approximate and model the dynamic breakthrough profiles obtained in this study. The profiles obtained at lower feed concentration were satisfactorily approximated by the pore diffusion model equation and the parameter, $N_{p^{\prime}}$, that gave a satisfactory fit was found to have a rounded off value of 2 . Dynamic breakthrough profiles obtained at higher feed concentrations were not amenable to approximation by the pore diffusion equation. Thus, it is conceivable that some other mechanisms in addition to pore diffusion are rate limiting. A possible explanation to this discrepancy maybe attributed to the relatively slow rate of adsorption to the matrix, as evidenced in the protein uptake profiles obtained in a finite medium.

\section{Conclusion}

In conclusion, the kinetics of adsorption of Igs onto r_PEZ in a finite medium can be described by the kinetic rate constant model. In the case of r_PEZ, our results suggest that the rate of adsorption of Ig to the matrix is more favorable than the rate of desorption. The mathematical equations that describe the pore diffusion model were used to model the dynamic breakthrough profiles. In the light of the analysis presented here, it appears that the mechanism of mass transfer in r_PEZ beads is limited by pore diffusion. In conjunction with the results presented in our earlier work (Subramanian and Sarkar [28, 31]), a set of engineering parameters are now available that can be used to scale up chromatographic separations based on r_PEZ. As most of the dynamic profiles obtained in this study were not satisfactorily fit using the pore and diffusion model, we will use the pulse injection techniques in conjunction with HETP equations to determine the various transport parameters relevant for scale-up, which will be a subject of a future publication.

\section{References}

[1] J.S. Bonnerjea, Oh, M. Hoare and P. Dunnhill, Biotechnology 4 (1986), p. 954.

[2] A.K. Hunter and G. Carta, J. Chromatogr. A 897 (2000), p. 65.

[3] A.K. Hunter and G. Carta, J. Chromatogr. A 897 (2000), p. 81.

[4] A.K. Hunter and G. Carta, J. Chromatogr. A 930 (2001), p. 79.

[5] A.M. Clausen, A. Subramanian and P.W. Carr, J. Chromatogr. A 831 (1999), p. 63.

[6] J. Nawrocki, C.J. Dunlap, P.W. Carr and J.A. Blackwell, Biotechnol. Prog. 10 (1994), p. 561.

[7] A. Subramanian, P.W. Carr and C.V. McNeff, J. Chromatogr. A 890 (2000), p. 15.

[8] M. Leonard, J. Chromatogr. A 699 (1997), p. 3.

[9] E. Boschetti, J. Chromatogr. 658 (1994), p. 207.

[10] P.R. Levison, R.M.H. Jones, D.W. Toome, S.E. Badger, M. Streater and N.D. Pathirana, J. Chromatogr. 734 (1996), p. 137.

[11] B.J. Horstmann, C.N. Kenny and H.A. Chase, J. Chomatogr. A 361 (1986), p. 179.

[12] A.E. Rodrigues, A.M.D. Ramos, J.M. Loureiro, M. Diaz and Z.P. Lu, Chem. Eng. Sci. 47 (1992), p. 4405.

[13] D.C. Nash and H.A. Chase, J. Chromatogr. A 807 (1998), p. 185.

[14] J. Horvath, E. Boschetti, L. Guerrier and N. Cooke, J. Chromatogr. A 679 (1994), p. 11.

[15] H.A. Chase, J. Chromatogr. 297 (1984), p. 179.

[16] G.L. Skidmore, B.J. Horstmann and H.A. Chase, J. Chomatogr. 498 (1990), p. 113.

[17] D.D. Frey, E. Schweinheim and C. Horvath, Biotechnol. Prog. 9 (1993), p. 273.

[18] G. Guiochon, S. Golshan Shirazi and A.M. Katti, Fundamentals of Preparative and Non-Linear Chromatography, Academic Press, New York (1994).

[19] B.J. Horstmann and H.A. Chase, Chem. Eng. Res. Des. 67 (1989), p. 243.

[20] F.H. Arnold, H.W. Blanch and C.R. Wilke, Chem. Eng. J. 30 (1985), p. B9.

[21] F.H. Arnold, J.J. Chalmers, M.S. Saunders, M.S. Croughan, H.W. Blanch and C.R. Wilke, Purif. Ferm. Prod. (1985), p. 113.

[22] A. Subramanian, J. Hommerding, J. Biochromatogr., submitted for publication.

[23] G. Carta, Chem. Eng. Sci. 43 (1988), p. 2877.

[24] G. Carta and A. Cincotti, Chem. Eng. Sci. 53 (1998), p. 3483.

[25] F.H. Arnold and H.W. Blanch, J. Chromatogr. 355 (1986), p. 13.

[26] A.M. Lenhoff, J. Chromatogr. 384 (1987), p. 285.

[27] V. Natarajan and S. Cramer, Sep. Sci. Tech. 35 (2000), p. 1719.

[28] A. Subramanian and S. Sarkar, J. Chromatogr. A 944 (2002), p. 179.

[29] S. Sarkar, Masters Thesis, University of Minnesota, Saint Paul, MN, 2002.

[30] J. Hommerding, Masters Thesis, University of Minnesota, Saint Paul, MN, 2001.

[31] A. Subramanian and S. Sarkar, J. Chromatogr. 989 (2003), p. 131. 